\title{
磷钼酸负载碳纳米管复合物的制备及其超级电容性能
}

\author{
郑 譞 ${ }^{1,2}$, 龚春丽 ${ }^{1}$, 刘 海 $^{1}$, 汪广进 ${ }^{1,2}$, 程 凡 ${ }^{1}$, 郑根稳 ${ }^{1}$, 文 胜 ${ }^{1}$, 熊传溪 ${ }^{2}$
}

(1. 湖北工程学院 化学与材料科学学院, 孝感 $432000 ; 2$. 武汉理工大学材料复合新技术国家重点实验室, 武汉 430070)

摘 要: 以聚多巴胺包覆碳纳米管为载体, 借助聚多巴胺超强的粘附性, 利用简单的溶液浸渍法制备了磷钼酸负载 碳纳米管(PMA@CNTs)复合物。通过傅里叶变换红外光谱(FTIR)、X 射线衍射 $(X R D) 、 X$ 射线光电子能谱(XPS)、 扫描电镜(SEM)、透射电镜(TEM)和电化学测试等对复合物的组成、结构、形态和超级电容性能进行了表征。结果 表明：聚多巴胺可将磷钼酸均匀且牢固地负载在碳纳米管上。在 $0.5 \mathrm{~mol} / \mathrm{L}$ 的 $\mathrm{H}_{2} \mathrm{SO}_{4}$ 电解液中, 复合物的最大比容 量为 $511.7 \mathrm{~F} / \mathrm{g}$, 最大能量密度可达 $66.8 \mathrm{Wh} / \mathrm{kg}$, 相应的功率密度为 $1000 \mathrm{~W} / \mathrm{kg}$ 。经过 1000 次循环, 比容量无任何衰 减。以上研究结果说明PMA@CNTs 复合物在电化学储能领域拥有极好的发展前景。

关 键 词: 聚多巴胺; 碳纳米管; 磷钼酸; 复合材料; 超级电容器

中图分类号: TQ174 文献标识码: A

\section{Preparation of Phosphomolybdic Acid Coated Carbon Nanotubes and Its Supercapacitive Properties}

\author{
ZHENG Xuan ${ }^{1,2}$, GONG Chun-Li ${ }^{1}$, LIU Hai ${ }^{1}$, WANG Guang-Jin ${ }^{1,2}$, CHENG Fan ${ }^{1}$, \\ ZHENG Gen-Wen ${ }^{1}$, WEN Sheng ${ }^{1}$, XIONG Chuan-Xi ${ }^{2}$
}

(1. College of Chemistry and Materials Science, Hubei Engineering University, Xiaogan 432000, China; 2. State Key Laboratory of Advanced Technology for Materials Synthesis and Processing, Wuhan University of Technology, Wuhan 430070, China)

\begin{abstract}
The phosphomolybdic acid coated carbon nanotubes (PMA@CNTs) were successfully fabricated by a facile polydopamine-assisted impregnation method, in which polydopamine can form an extraordinary adhesive interlayer to homogeneously adhere PMA on the surfaces of CNTs. The composition, structure, morphology and supercapacitive performances of the resulting PMA@CNTs hybrids were systematically characterized by a range of experimental tools including fourier transform infrared spectrometer (FTIR), X-ray diffraction (XRD), X-ray photoelectron spectroscope(XPS), scanning electron microscope(SEM), transmission electron microscope(TEM), cyclic voltammetry (CV) and galvanostatic charge/discharge (GCD). The PMA can homogeneously loaded onto the surface of the CNTs with the aid of superior adhesion of polydopamine. Here, the performance of the resulting PMA@CNTs hybrids as supercapacitor electrodes was investigated in a three-electrode arrangement using an aqueous electrolyte $\left(0.5 \mathrm{~mol} / \mathrm{L} \mathrm{H}_{2} \mathrm{SO}_{4}\right)$. The supercapacitor assembled with the PMA $\mathrm{P}_{50} @ \mathrm{CNTs}$ hybrids exhibit the highest specific capacitances $(511.7 \mathrm{~F} / \mathrm{g}$ at $10 \mathrm{mV} / \mathrm{s})$ and maximum energy density of $66.8 \mathrm{Wh} / \mathrm{kg}$ at power density of $1000 \mathrm{~W} / \mathrm{kg}$, based on the total mass of active materials. In addition, the supercapacitor also has excellent cycling
\end{abstract}

收稿日期: 2012-05-30; 收到修改稿日期：2012-09-12

基金项目：国家自然科学基金青年基金(51303048，51403058); 湖北省自然科学基金(2015CFC769); 中央校基本科研业务 (2016-YB-011)

National Natural Science Foundation--Youth Foundation (51303048, 51403058); Natural Science Foundation of Hubei Province (2015CFC769); Fundamental Research Funds for the Central Universities (2016-YB-011)

作者简介: 郑 譞(1988-), 男, 博士研究生, 实验师. E-mail: 63474559@qq.com

通讯作者: 文 胜, 副教授. E-mail: shengwen_hbeu@163.com 
stability retaining $>100 \%$ of its specific capacitances after 1000 cycles at current density of $5 \mathrm{~A} / \mathrm{g}$. These results demonstrate a simple and scalable application of PMA@CNTs hybrids toward electrochemical energy storage.

Key words: polydopamine; carbon nanotubes; phosphomolybdic acid; hybrids supercapacitors

杂多酸(Heteropoly acids, HPAs)属于多金属含氧 酸盐, 具有良好的电子和质子传输和储存能力(可接 收多达 32 个电子)、特殊的光还原性、较高的 “晶格 氧” 活泼性和高的质子酸性等独特的物理化学性质, 已引起广泛关注 ${ }^{[1-5]}$ 。以最常见的 Keggin 型杂多酸 $\left(\mathrm{Hn}\left\{\mathrm{XM}_{12} \mathrm{O}_{40}\right\} \cdot \mathrm{mH}_{2} \mathrm{O}\right)$ 为例, 由于其拥有确定且易简 单调控的结构使得杂多酸在催化 ${ }^{[6-8]}$ 、医药 ${ }^{[9-12]}$ 和电 显色装置 ${ }^{[13-15]}$ 等方面具有广泛应用。但是, 杂多酸存 在比表面积小、易溶于极性溶剂等缺点, 难以发挥其 作为固体酸在催化和电化学领域中的优势 ${ }^{[16-18]}$ 。

为了克服上述缺点, 可以将杂多酸均匀分散或 负载在具有较大比表面积的纳米材料表面。碳纳米 管(Carbon nanotubes, CNTs)具有超高的比表面积、优 异的导电性、化学稳定性和机械性能, 是杂多酸负载 基体的首选材料 ${ }^{[19-21]}$ 。然而, 纯碳纳米管的表面活性 位点较少, 抑制了杂多酸的负载量, 在实际应用中 多采用强酸化处理来增加碳管表面的功能基团 ${ }^{[22-24]}$, 但强酸处理会严重破坏碳纳米管的结构, 使其物理 性能降低。有研究人员采用季铵盐型超支化聚合物 作为桥连剂将杂多酸固定在酸化碳纳米管表面形成 新型阳极材料 ${ }^{[25]}$, 但是制备过程复杂, 需要一系列 修饰步骤来提高杂多酸与碳纳米管之间的相容性来 抵消酸化过程对碳纳米管性能的影响, 应用成本较 高。聚多巴胺(Polydopamine, PDA) 是贝壳、蚌等生物 分泌的粘性蛋白的主要成分，几乎能粘附在任何基 体材料表面, 并在粘附基体以后还能够作为粘附其 它物质的载体, 这为二次改性提供了一种通用普适 性的途径 ${ }^{[26-29]}$ 。利用聚多巴胺结构中酚羟基和类吲 哚官能团的超强吸附性 ${ }^{[30]}$, 可以避免强酸氧化过程 对碳管结构造成的损伤, 而且它对杂多酸阴离子的 吸附作用可以将杂多酸固定在基体表面。

针对常规方法制备杂多酸负载碳纳米管存在工 艺复杂、碳管结构易损伤的缺点, 本工作以未经酸化 处理的原始碳纳米管为原料, 通过多巴胺的氧化自 聚合在碳纳米管表面原位形成聚多巴胺包覆层, 再 以聚多巴胺包覆碳纳米管( PDA-CNTs) 作为载体, 利 用聚多巴胺超强的粘附性将 Keggin 型杂多酸中较常 用的磷钼酸 $\left(\mathrm{H}_{3}\left[\mathrm{P}\left(\mathrm{Mo}_{3} \mathrm{O}_{10}\right)_{4}\right]\right.$, PMA $)$ 负载于 PDACNTs 表面得到磷钼酸负载碳纳米管(PMA@CNTs) 复合物, 并对其组成、结构和形态进行了表征, 再利 用传统三电极体系对合成 PMA@CNTs 复合物的超
级电容性能进行分析。

\section{1 实验方法}

\section{1 试剂与仪器}

多壁碳纳米管: 纯度> 95\%, 平均直径 10 15 nm (中国科学院成都有机化学有限公司); 三(羟甲基)氨 基甲烷盐酸盐(Tris-HCl): 纯度 $\geqslant 99 \%$, 阿拉丁化学 试剂有限公司; 多巴胺: 纯度 $>99 \%$, 西格玛阿尔德 里奇化学试剂有限公司; 磷钼酸: 纯度 $\geqslant 99.7 \%$, 阿 拉丁化学试剂有限公司。

Chi660E 电化学工作站(上海辰华仪器有限公司); JSM-7100F 扫描电子显微镜(日本 JEOL 公司); Tecnai G20 透射电子显微镜(美国 FEI 公司); ESCALAB 250Xi XPS 光电子能谱仪(英国 Thermo Scientific 公 司); D8 Advance X 射线衍射仪(德国 Bruker 公司); Nexus 红外光谱仪(美国 Nicolet 公司); STA449c 同步 热分析仪(德国 Netzsch 公司)。

\subsection{PMA@CNTs 复合物的制备}

PMA@CNTs 复合物的制备过程如图 1 所示, 首 先利用多巴胺的氧化自聚合制备出 PDA-CNTs, 再 通过溶液浸渍法得到PMA@CNTs 产物, 具体步骤如 下: 称取 $20 \mathrm{mg}$ 多巴胺, 加入到 $100 \mathrm{~mL}$ Tris- $\mathrm{HCl}$ 缓冲 溶液 $(\mathrm{pH}=8.5)$ 中, 在 $25^{\circ} \mathrm{C}$ 下磁力摚拌 $30 \mathrm{~min}$, 称取 $20 \mathrm{mg}$ 的多壁碳纳米管加入上述溶液中并继续室温摚 拌 $24 \mathrm{~h}$, 产物经抽滤、去离子水反复洗涤、干燥后得到 PDA-CNTs。将 $10 \mathrm{mg}$ PDA-CNTs 分别加入到 0.4、2、 $10 、 50 \mathrm{mmol} / \mathrm{L}$ 的磷钿酸水溶液 $(100 \mathrm{~mL})$ 中, 超声分散 $1 \mathrm{~h}$ 后, 室温下继续搅拌 $48 \mathrm{~h}$ 后, 经真空抽滤、洗涤、 干燥后即可得到 PMA@CNTs, 其中 $\mathrm{PMA}_{0.4} @ \mathrm{CNTs}$ 、 PMA $@$ @ CNTs、PMA $10 @ C N T s$ 和 PMA 50 @ CNTs 分别 对应上述 4 种浓度磷钼酸水溶液所得到的 PMA@CNTs。

\subsection{PMA@CNTs 复合物电极的制备及其超级 电容性能表征}

将PMA@CNTs 复合物、导电剂(乙炔黑)以及粘 结剂聚偏氟乙烯(PVDF)按质量比 8:1:1 混合, 滴入 适量 N-甲基吡咯烷酮(NMP)制备成料浆，并利用微 量移液器将制备料浆涂敷在玻璃碳电极表面, 然后 将涂敷料浆的玻璃碳电极放入 $60^{\circ} \mathrm{C}$ 的真空烘箱 $24 \mathrm{~h}$ 


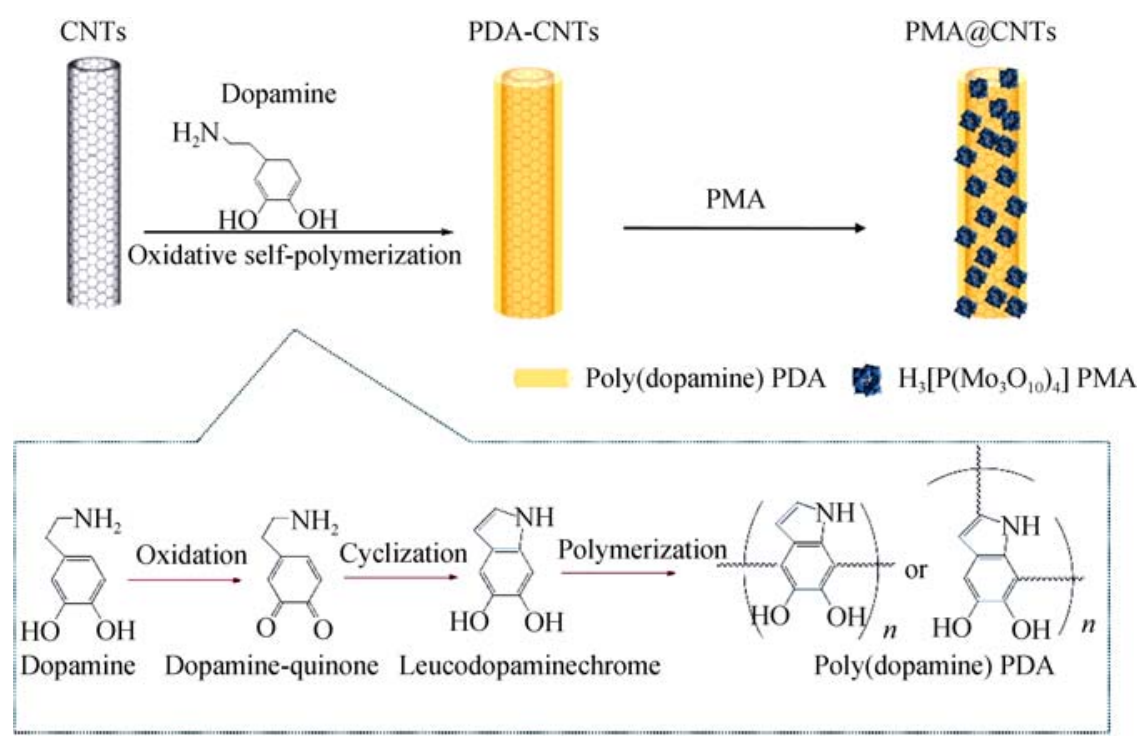

图 1 多巴胺氧化自聚合机理 ${ }^{[25-27]}$ 及 PMA@CNTs 复合物的制备过程示意图

Fig. 1 Illustration of oxidative self-polymerization mechanism of dopamine ${ }^{[25-27]}$ and preparation procedure of PMA@CNTs hybrids

烘干，用作工作电极，料浆中活性物质占 $80 \%$ 。再将 烘干的工作电极浸入 $0.5 \mathrm{~mol} / \mathrm{L}$ 的 $\mathrm{H}_{2} \mathrm{SO}_{4}$ 电解质溶液 中活化 $5 \mathrm{~h}$ 。借助 Chi 660E 型电化学工作站对合成复 合物的超级电容性能进行分析, 并根据公式(1)和公 式(2)计算电极材料的比容量 ${ }^{[31]}$, 其中 $C$ 为比容量 $(\mathrm{F} / \mathrm{g}), s$ 为扫描速率 $(\mathrm{V} / \mathrm{s}), m$ 为活性物质质量 $(\mathrm{g}), \Delta V$ 为电化学窗口 $(\mathrm{V}), I_{1}$ 为循环伏安响应电流 $(\mathrm{A}), \Delta t$ 为 放电时间(s), $I_{2}$ 为充放电电流(A)。

$$
\begin{aligned}
& C=\frac{\int I_{1} \mathrm{~d} V}{s m \Delta V} \\
& C=\frac{I_{2} \Delta t}{m \Delta V}
\end{aligned}
$$

能量密度 $(E)$ 和功率密度 $(P)$ 分别由公式(3)和(4) 计算 ${ }^{[32]}$, 其中, $C$ 是根据公式(2)计算的比容量 $(\mathrm{F} / \mathrm{g})$, $\Delta V$ 为电化学窗口 $(\mathrm{V}), \Delta t$ 为放电时间 $(\mathrm{s})$ 。

$$
\begin{gathered}
E=0.5 C \Delta V^{2} / 3.6 \\
P=\frac{E \times 3600}{\Delta t}
\end{gathered}
$$

\section{2 结果与讨论}

\subsection{PMA@CNTs 复合物的组成和结构}

图 2 为 $\mathrm{CNTs} 、 \mathrm{PDA}-\mathrm{CNTs}$ 和 $\mathrm{PMA}_{50} @ \mathrm{CNTs}$ 的 FTIR 对比图, 从图中可以看出, 与纯 CNTs 相比, PDA-CNTs 和 PMA $50 @$ CNTs 在 1692、1512、1407 cm ${ }^{-1}$ 附近有较明显吸收峰, 其中 $1692 \mathrm{~cm}^{-1}$ 为 $\mathrm{C}=\mathrm{O}$ 吸收峰, $1512 \mathrm{~cm}^{-1}$ 对应苯环上的 $\mathrm{C}=\mathrm{C}$ 伸缩振动吸收峰, $1407 \mathrm{~cm}^{-1}$ 对应 $\mathrm{C}-\mathrm{N}$ 伸缩振动峰, 以上三种特征峰与聚多巴胺 (PDA)的分子结构特征相符。而在 $\mathrm{PMA}_{50} @ \mathrm{CNTs}$ 的
谱图中, 还可见磷钿酸在 $1084 \mathrm{~cm}^{-1}(\mathrm{P}-\mathrm{O}) 、 986 \mathrm{~cm}^{-1}$ (Mo-O) 和 $884 \mathrm{~cm}^{-1}$ (Mo-O-Mo) 附近的吸收峰, 但这 几个吸收峰的峰强度较弱, 且峰位置均有部分蓝移, 这可能是由于 $\mathrm{PMA}_{50} @ \mathrm{CNTs}$ 中磷钿酸含量较低以及 磷钿酸阴离子与聚多巴胺中的功能基团相结合导致 各吸收峰减弱 ${ }^{[33]}$ 。

从 XRD 图谱可以清楚反映出碳纳米管在包覆前 后的晶型结构变化, 如图 3 所示, CNTs 样品只在 $2 \theta=$ $26.1^{\circ}$ 和 $43.2^{\circ}$ 有两个宽峰, 对应(002)和(100)晶面的 衍射。经聚多巴胺包覆处理之后, PDA-CNTs 样品的 XRD 图谱中并未出现新的衍射峰, 这说明原位聚合 形成的 PDA 为无定形结构。而对于 $\mathrm{PMA}_{50} @ \mathrm{CNTs}$ 样品, 其衍射图谱与 CNTs 和 PDA-CNTs 相比有了明 显的变化, 在 $2 \theta=10.8^{\circ} 、 15.3^{\circ} 、 21.7^{\circ} 、 26.6^{\circ} 、 30.9^{\circ}$ 、 $36.4^{\circ}$ 和 $39.7^{\circ}$ 处出现了新的尖锐衍射峰, 对比标准 PDF 卡片, 归属于磷钼酸(PDF 38-0179)的(210)、 (311)、(214)、(430)、(043)、(516)和(046)晶面的特

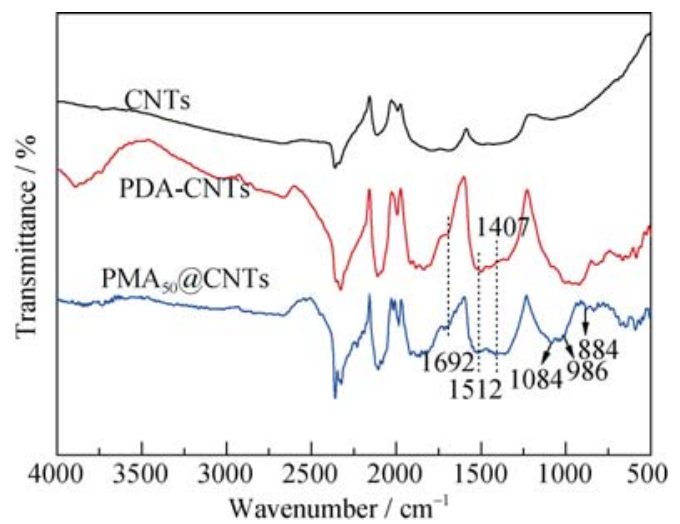

图 2 CNTs、PDA-CNTs 和 PMA $\mathrm{A}_{50} @ \mathrm{CNTs}$ 的 FTIR 谱图

Fig. 2 FTIR spectra of CNTs, PDA-CNTs and PMA POCNTs 


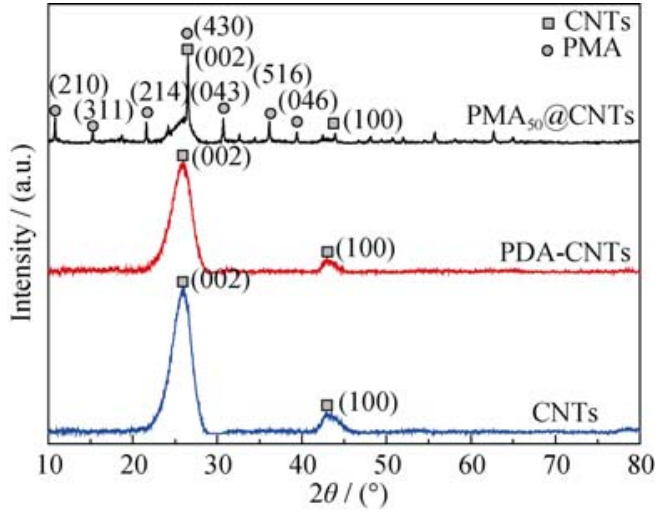

图 3 CNTs、PDA-CNTs 和 $\mathrm{PMA}_{50} @ \mathrm{CNTs}$ 的 XRD 图谱 Fig. 3 XRD patterns of CNTs, PDA-CNTs and PMA ${ }_{50} @$ CNTs

征衍射峰, 这充分表明磷钿酸已经成功负载在碳纳 米管上。

磷钿酸负载在聚多巴胺包覆的碳纳米管表面同 样也能被 XPS 谱图(图 4)证实, 如图 4(a)宽谱扫描所 示, $\mathrm{PMA}_{50} @ \mathrm{CNTs}$ 复合物含有 4 个元素信号峰: $\mathrm{C} 1 \mathrm{~s}$ 、 $\mathrm{N} 1 \mathrm{~s} 、 \mathrm{O} 1 \mathrm{~s}$ 和 Mo3d, 其中 $\mathrm{C} 1 \mathrm{~s}$ 经分峰拟合处理可以分 成四个峰(图 4(b)), 键能 (Binding Energy) 分别为 $284.8,285.6,286.4$ 和 $287.8 \mathrm{eV}$, 分别对应着 $\mathrm{C}-\mathrm{C}$, $\mathrm{C}-\mathrm{N}, \mathrm{C}-\mathrm{O}, \mathrm{C}=\mathrm{O}$ 中碳原子的谱峰, 其中 $285.6 \mathrm{eV}$ 来 自于聚多巴胺中的 $\mathrm{C}-\mathrm{N}$ 。图 4(c)中的 $\mathrm{N} 1 \mathrm{~s}$ 可以分为 两个峰: 398.7 和 $400.4 \mathrm{eV}$, 分别对应 $-\mathrm{N}=$ 和 $\mathrm{N}-\mathrm{H}$, $\mathrm{N}-\mathrm{H}$ 来自于 PDA 中的氨基官能团, $-\mathrm{N}=$ 来自于多巴 胺氧化过程中的结构演化(见图 1 多巴胺氧化自聚机 理)，这也进一步证实聚多巴胺中间包覆层的存在 ${ }^{[34]}$ 。 另外, 结合图 4(d)中 Mo3d 的分峰拟合图可知, Mo 的 $3 \mathrm{~d}_{5 / 2}$ 和 $3 \mathrm{~d}_{3 / 2}$ 特征双峰分别出现在键能为 $232.9 \mathrm{eV}$ 和 $236.1 \mathrm{eV}$ 处, 这与其在磷钼酸中的价态一致, 证明 Mo3d 来自于磷钼酸中的 $\mathrm{Mo}^{[35]}$ 。

结合 SEM 与 TEM 进一步对 PMA ${ }_{50} @ \mathrm{CNTs}$ 复合 物的结构进行表征。图 5(a)和 5(b) 分别为 $\mathrm{PMA}_{50} @ \mathrm{CNTs}$ 的 SEM 照片和 EDX 图谱, 可以看出, $\mathrm{PMA}_{50} @ \mathrm{CNTs}$ 的平均直径约在 15 20 nm 之间, 相比 于原始 CNTs(平均直径 10 15 nm) 略有增加, 外侧管 壁有一层薄薄的高分子膜, 膜厚约 $2.5 \mathrm{~nm}$ (图 5(d)), 调节多巴胺的浓度 $(2 \mathrm{~g} / \mathrm{L})$ 和溶液 $\mathrm{pH}(\mathrm{pH}=8.5)$ 后, 并在 室温搅拌一段时间, 可以使 PMA 在 CNTs 表面发生 氧化自聚合生成一层聚多巴胺(PDA)膜将 CNTs 包覆 起来 ${ }^{[36]}$ 。其次，图 5(b) 的能谱分析可以更直观证明磷 钼酸负载在聚多巴胺包覆的碳纳米管上, 图中除 $\mathrm{C}$ 元素外还含有 $N 、 O 、 M o 、 P$ 四种元素, $N$ 元素只可 能来自于聚多巴胺, 而 $\mathrm{O}$ 元素既可能存在于聚多巴 胺中, 又可能存在于磷钿酸中, $\mathrm{Mo}$ 和 $\mathrm{P}$ 的存在则充分
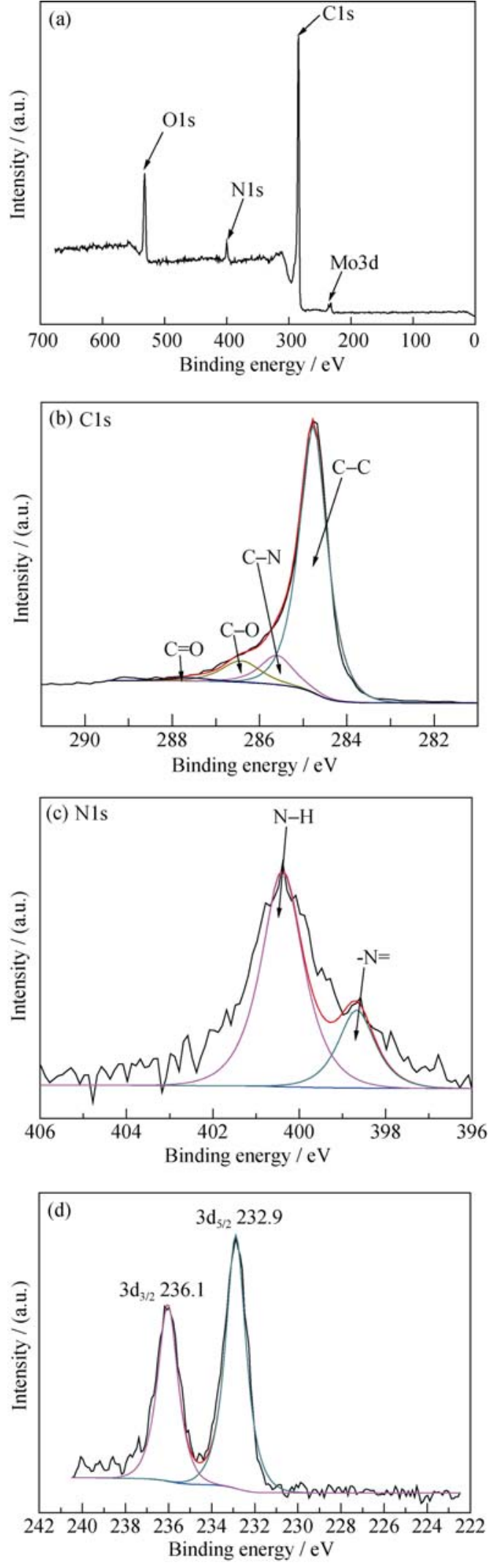

图 $4 \mathrm{PMA}_{50} @ \mathrm{CNTs}$ 的(a)XPS 宽谱扫描图, (b) $\mathrm{C} 1 \mathrm{~s}$ 分峰图, (c)N1s 分峰图, (d)Mo3d 分峰图

Fig. 4 XPS spectra of PMA ${ }_{50} @$ CNTs: (a) wide scan; peak deconvolution of (b) C1s, (c) N1s, and (d) Mo3d

说明磷钼酸成功负载于 PDA-CNTs 上。最后, 从表 1 的 EDX 元素统计结果可以发现 P 元素含量最低, 这 


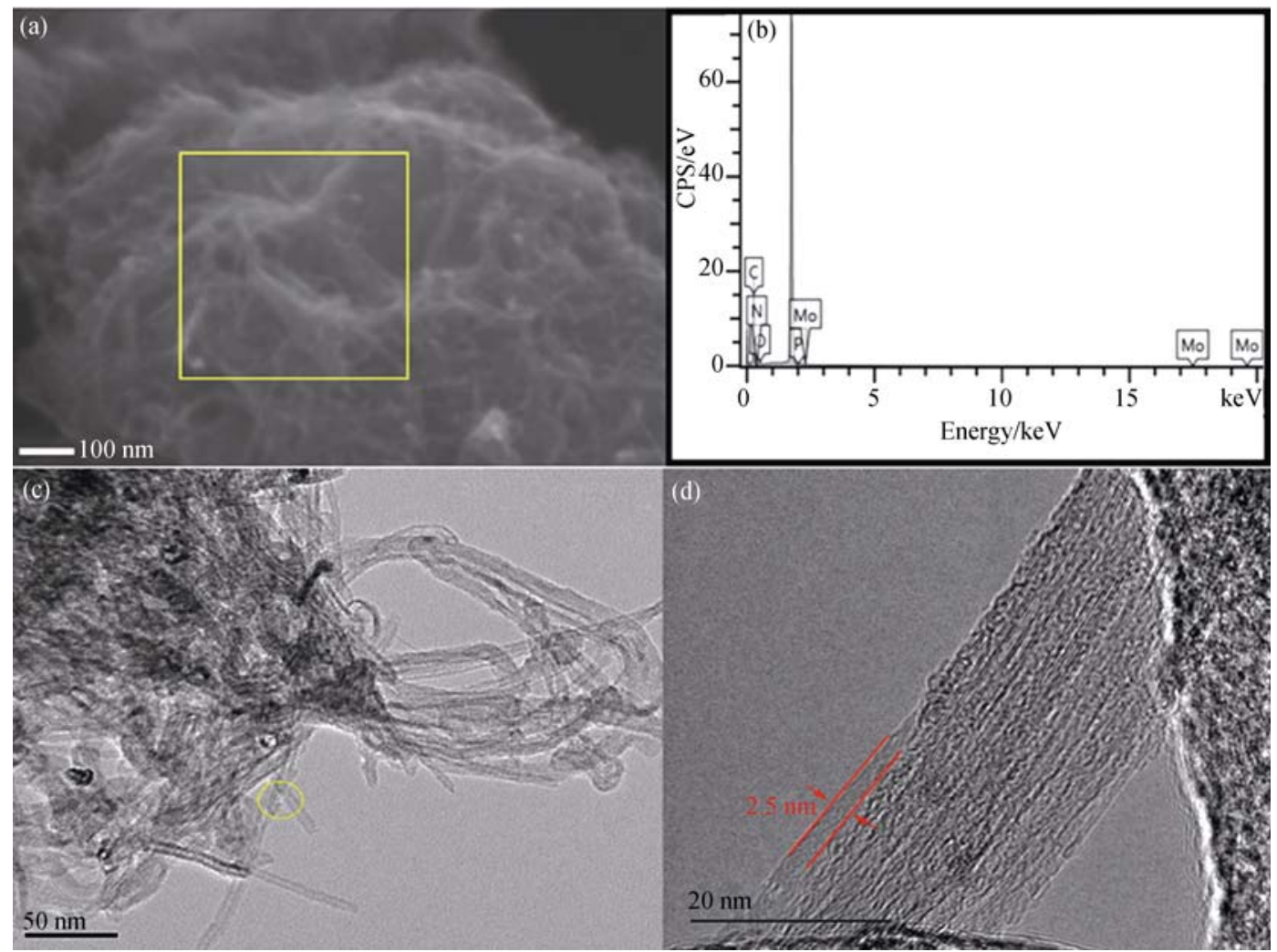

图 5 (a)PMA $\mathrm{PM}_{50} @ \mathrm{CNTs}$ 的 SEM 照片, (b)PMA 50 @CNTs 的 EDX 图谱, (c)、(d)PMA $\mathrm{PM}_{50} @ \mathrm{CNTs}$ 的 TEM 照片

Fig. 5 SEM images of PMA 50 @CNTs (a), EDXspectra of PMA ${ }_{50} @$ CNTs (b), and TEM images of PMA ${ }_{50} @ C$ Ts (c), (d)

与磷钼酸 $\left(\mathrm{H}_{3}\left[\mathrm{P}\left(\mathrm{Mo}_{3} \mathrm{O}_{10}\right)_{4}\right]\right)$ 中自身 $\mathrm{P}$ 比例较低有关。 值得注意的是, 与图 5(b)的 EDX 能谱图对比发现, 图 4(a)所反映的元素信号峰中未出现 P 元素, 这一方 面是因为复合材料中磷钼酸的含量较低(如表 1 所示), 而磷钼酸中磷元素本身又占比很低, 因此检测不到 $\mathrm{P}$ 的信号峰; 另一方面与 XPS 本身的探测深度有关: 依据文献[37], XPS 对有机高分子的测量厚度约为 $7.5 \mathrm{~nm}$, 导致 P 的信号峰不易被探测到。

图 6 是 CNTs、PDA-CNTs 和 $\mathrm{PMA}_{50} @ \mathrm{CNTs}$ 在 空气气氛下的 TGA 曲线, 可以看出 CNTs 的失重起 始于 $350^{\circ} \mathrm{C}$, 在 $560^{\circ} \mathrm{C}$ 基本分解完全, 这主要来源于 碳纳米管在空气气氛下的热氧化分解 ${ }^{[35]}$ 。而 PDA-CNTs

表 1 PMA $50 @ C N T s$ 的 EDX 元素组成

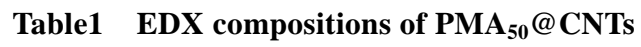

\begin{tabular}{ccc}
\hline Element & at/\% & wt $/ \%$ \\
\hline $\mathrm{C}$ & 82.99 & 79.93 \\
$\mathrm{~N}$ & 3.73 & 5.96 \\
$\mathrm{O}$ & 7.05 & 11.37 \\
$\mathrm{P}$ & 1.86 & 0.86 \\
$\mathrm{M}_{\mathrm{O}}$ & 2.19 & \\
Total & 100.00 & \\
\hline
\end{tabular}

和 $\mathrm{PMA}_{50} @ \mathrm{CNTs}$ 的失重开始于 $200^{\circ} \mathrm{C}$, 这是由于 PDA 分子主链的热分解所致, 这一阶段持续到 $450^{\circ} \mathrm{C}$ 后 PDA 完全分解，重量损失约 $25 \%$; 紧接着开始第 二阶段碳纳米管的热氧化分解, 在 $560^{\circ} \mathrm{C}$ 以上碳纳米 管也分解完全。值得注意的是, $\mathrm{PMA}_{50} @ \mathrm{CNTs}$ 相比于 PDA-CNTs 而言在 $560^{\circ} \mathrm{C}$ 之后的平台区为磷钿酸 ${ }^{[38]}$, 重量百分比约为 $3.6 \mathrm{wt} \%$, 这一结果与 $\mathrm{EDX}$ 中由 $\mathrm{Mo}$ 含量所计算出的磷钼酸含量基本一致。

\subsection{PMA@CNTs 复合物的超级电容性能测试} 为进一步证实 PMA@CNTs 复合物的超级电容特征,

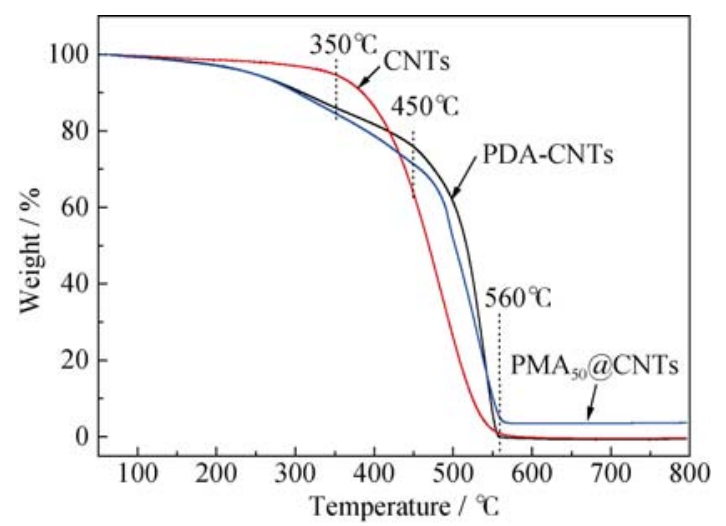

图 6 CNTs、PDA-CNTs 和 $\mathrm{PMA}_{50} @ \mathrm{CNTs}$ 的 TGA 图谱 Fig.6 TGA curves of CNTs, PDA-CNTs and PMA $50 @$ CNTs 
首先利用传统三电极体系分别对 PDA-CNTs、 $\mathrm{PMA}_{0.4} @ \mathrm{CNTs}$ 、 $\mathrm{PMA}_{2} @ \mathrm{CNTs}$ 、PMA $10 @ \mathrm{CNTs}$ 和 $\mathrm{PMA}_{50} @ \mathrm{CNTs}$ 电极材料进行循环伏安测试, 扫速为 $10 \mathrm{mV} / \mathrm{s}$, 如图 7(a)所示。从图 7(a)可以看出, 相比于 PDA-CNTs 基本类似于矩形的 CV 曲线, PMA@ CNTs 复合物在 $0.2 \sim 0.6 \mathrm{~V}$ 之间出现明显氧化还原 电流峰。这些氧化还原峰都可归属于 PMA 杂多阴 离子在 $0.5 \mathrm{~mol} / \mathrm{L} \mathrm{H}_{2} \mathrm{SO}_{4}$ 中的氧化还原过程, 且随着 磷钼酸水溶液浓度升高, 制备得到的PMA@CNTs 的 氧化还原峰值明显增加, 直至 $\mathrm{PMA}_{50} @ \mathrm{CNTs}$ 达到最 大, 说明 PMA 可以极大地增强 PMA@CNTs 材料的 电化学活性。图 7(b)显示了上述 5 种电容器在 $2 \mathrm{~A} / \mathrm{g}$ 电流密度下的恒流充放电曲线, 则可进一步说明伴 随磷钼酸水溶液浓度增加制备得到的PMA@CNTs, 其比容量值也随之大幅增加, 这是因为其容量不仅 包含 PDA-CNTs 的双电层电容, 而且极大部分来自 PMA 贡献的法拉第赝电容 ${ }^{[39]}$ 。

基于图 7(a)、(b)研究结果, 我们在不同扫速和电 流密度下对 $\mathrm{PMA}_{50} @ \mathrm{CNTs}$ 电极材料进行了循环伏安
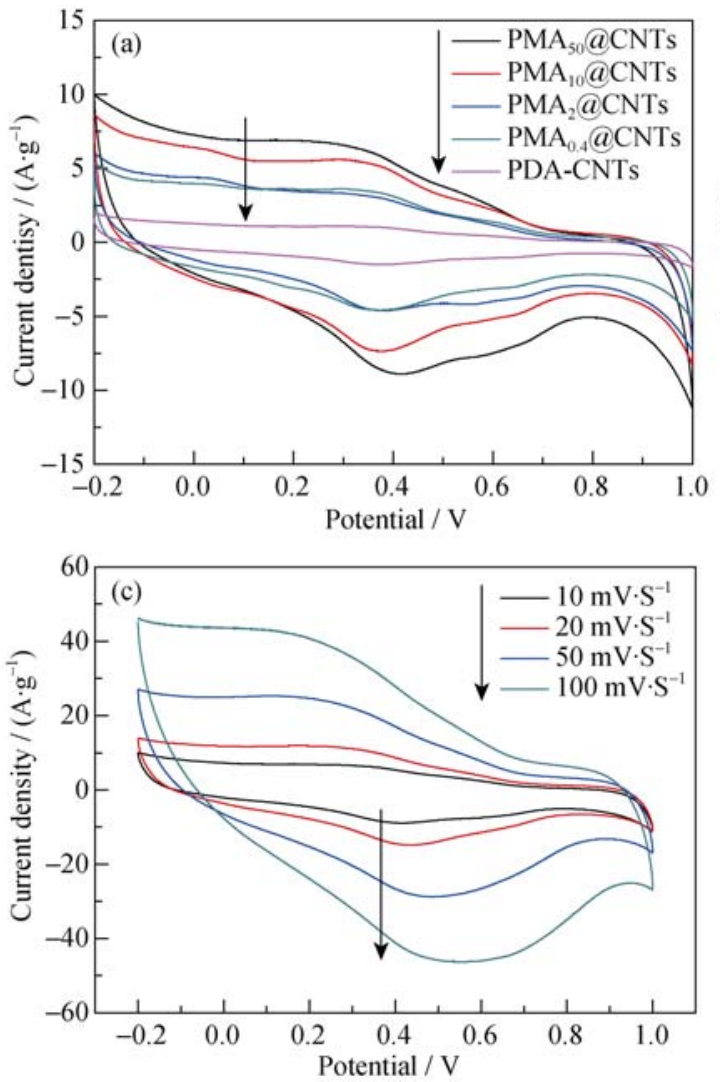

和恒流充放电测试，结果见图 7(c)和(d)。从图 7(c)可 以看出, $\mathrm{PMA}_{50} @ \mathrm{CNTs}$ 的氧化还原峰电流随着扫速 的增加而明显增大，同时充放电曲线(图 7(d))具有很 好的线性和对称性，说明 $\mathrm{PMA}_{50} @ \mathrm{CNTs}$ 电极材料具 有理想的电容特性。

根据公式(1)和(2)分别计算 $\mathrm{PMA}_{50} @ \mathrm{CNTs}$ 在不 同扫速和电流密度下的比容量, 如图 8(a)所示。由图 8 (a)可知, 扫速为 $10 \mathrm{mV} / \mathrm{s}$ 时, $\mathrm{PMA}_{50} @ \mathrm{CNTs}$ 的比容 量高达 $511.7 \mathrm{~F} / \mathrm{g}$, 但当扫速增至 $100 \mathrm{mV} / \mathrm{s}$ 时, $\mathrm{PMA}_{50} @ \mathrm{CNTs}$ 的比容量降至 $276.3 \mathrm{~F} / \mathrm{g}$, 容量保持率 为 $54 \%$ 。与此同时，当电流密度增加至 $20 \mathrm{~A} / \mathrm{g}$ 时， $\mathrm{PMA}_{50} @ \mathrm{CNTs}$ 的比容量仍具有 $312 \mathrm{~F} / \mathrm{g}$, 容量保持率 为 $64.9 \%$, 以上结果说明 $\mathrm{PMA}_{50} @ \mathrm{CNTs}$ 的比容量高, 倍率性能较好, 适合用作超级电容电极材料。

图 8(b)给出了 $\mathrm{PMA}_{50} @ \mathrm{CNTs}$ 复合物在 $5 \mathrm{~A} / \mathrm{g}$ 电 流密度下, 循环 1000 次的容量保持率曲线。由于电 极材料的前期活化过程，在前 400 次循环呈现上升 趋势。而循环 1000 次后, PMA ${ }_{50} @ \mathrm{CNTs}$ 的容量不仅无 任何衰减, 反而有 $2 \%$ 的增加, 原因可能是由于反复
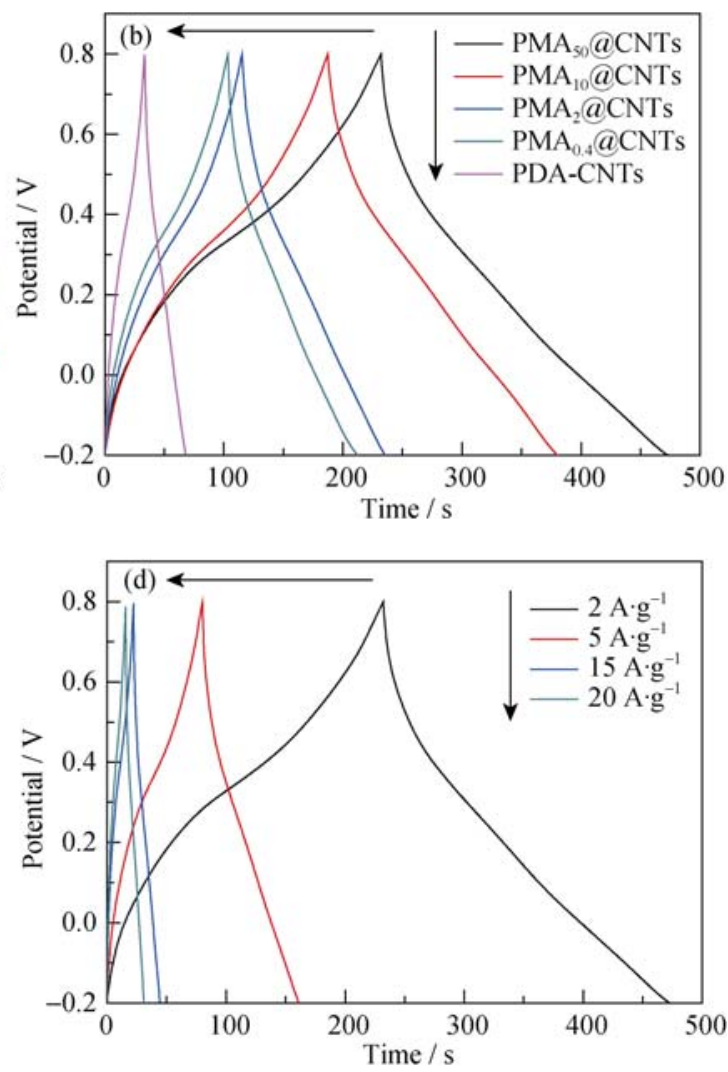

图 7 PDA-CNTs、PMA $0.4 @ C N T s 、 P M A_{2} @ C N T s 、 P M A_{10} @ C N T s$ 和 PMA $50 @ C N T s$ 在 10 mV/s 扫速下的循环伏安曲线(a)和在 2 A/g 电流密度下的恒流充放电曲线(b)以及 $\mathrm{PMA}_{50} @ \mathrm{CNTs}$ 在不同扫速下的循环伏安曲线(c), 不同电流密度下的恒流充放电曲线(d)

Fig. 7 (a) Cyclic voltammograms at scan rate of $10 \mathrm{mV} / \mathrm{s}$ ) and (b) galvanostatic charge-dischargecyclic curves at current density of $2 \mathrm{~A} / \mathrm{g}$ for PDA-CNTs, PMA ${ }_{0.4} @$ CNTs, PMA $@$ CNTs, PMA $10 @$ CNTs andPMA 50 CNTs coin cell, and (c) cyclic voltammograms at different scan rates and (d) charge/discharge curves at different current densities for PMA $\mathrm{A}_{50} @ \mathrm{CNTs}$ 

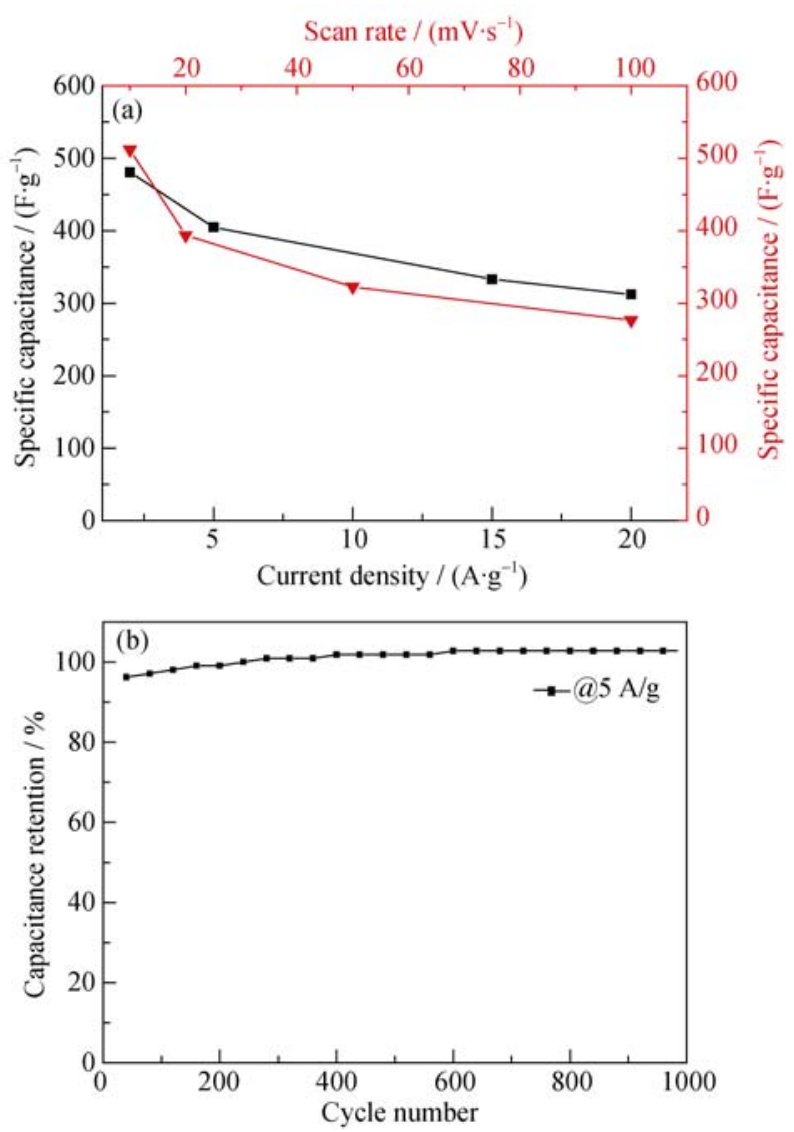

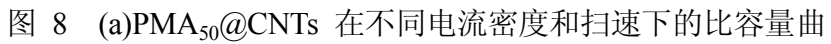
线和(b)PMA $\mathrm{PM}_{50}$ CNTs 在 $5 \mathrm{~A} / \mathrm{g}$ 电流密度下的循环稳定性测试 结果

Fig. 8 (a) Specific capacitance of the PMA ${ }_{50} @ \mathrm{CNT}$ selectrode as a function of scan rate and current density and (b) cycling stability of the PMA 50 CNTs electrode at a current density of $5 \mathrm{~A} / \mathrm{g}$

充放电过程促进了电极材料获取更多离子的能力 ${ }^{[40]}$ 。 由此可见, $\mathrm{PMA}_{50} @ \mathrm{CNTs}$ 复合物是一种新型高性能 的超级电容器电极材料。

为了更综合的评估 $\mathrm{PMA}_{50} @ \mathrm{CNTs}$ 复合物电极 材料的电化学性能，根据公式(3)和(4)可以计算得 到能量密度和功率密度之间的关系, 计算结果如图 9 所示, 由图可知, 以 $0.5 \mathrm{~mol} / \mathrm{L} \mathrm{H}_{2} \mathrm{SO}_{4}$ 为电解质的 $\mathrm{PMA}_{50} @ \mathrm{CNTs}$ 复合物电极材料在 $2 \mathrm{~A} / \mathrm{g}$ 电流密度下的 最大能量密度可达 $66.8 \mathrm{Wh} / \mathrm{kg}$, 对应的功率密度为 $1000 \mathrm{~W} / \mathrm{kg}$; 当功率密度为 $9992 \mathrm{~W} / \mathrm{kg}$ 时, 能量密度仍 有 $44.4 \mathrm{Wh} / \mathrm{kg}$ 。这意味着利用 $\mathrm{PMA}_{50} @ \mathrm{CNTs}$ 复合物 为电极材料制作超级电容电容器, 在一定程度上其能 量和功率方面将会高于铅酸电池 $(25 \sim 30 \mathrm{Wh} / \mathrm{kg})$, 且 与镍氢电池 $(60 \sim 65 \mathrm{Wh} / \mathrm{kg})$ 相当, 但仍远低于锂电池 ( $>80 \mathrm{Wh} / \mathrm{kg})$ 。但与现有商业碳材料(5 6 Wh/kg)和已 报到氮掺杂有序多孔碳材料 $(63 \mathrm{Wh} / \mathrm{kg})$ 相比 ${ }^{[41]}$, $\mathrm{PMA}_{50} @ \mathrm{CNTs}$ 复合物有望成为下一代高性能超级电 容器的理想电极材料。

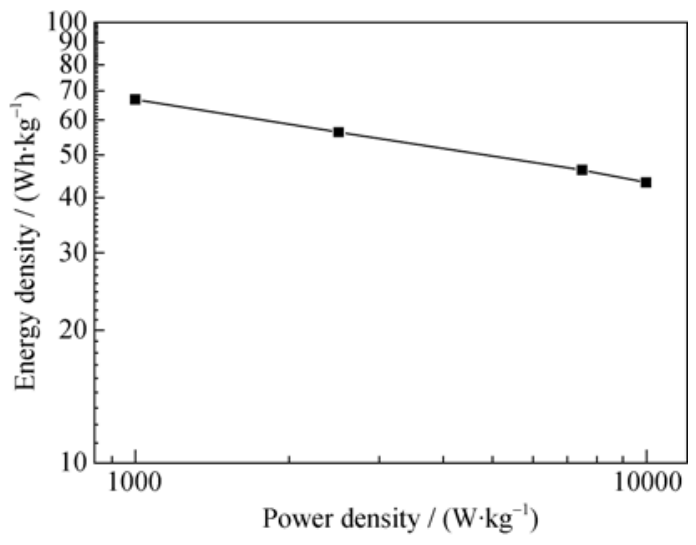

图 $9 \mathrm{PMA}_{50} @ \mathrm{CNTs}$ 电极材料的功率密度与能量密度的关系 曲线

Fig. 9 Ragone plot of $\mathrm{PMA}_{50} @$ CNTs electrodes

\section{3 结论}

研究以聚多巴胺包覆碳纳米管(PDA-CNTs)作为 载体, 选用磷钼酸(PMA)作为原料, 并采用浸渍法利 用 PDA 中的酚羟基和类吲哚官能团对 PMA 的吸附 性将 PMA 负载于 PDA-CNTs 表面制备得到磷钼酸负 载碳纳米管(PMA@CNTs)复合物。FTIR、XRD、XPS、 SEM、TEM 和 TGA 表征结果表明实验成功制备了 PMA@CNTs 复合物。在 $0.5 \mathrm{~mol} / \mathrm{L}$ 的 $\mathrm{H}_{2} \mathrm{SO}_{4}$ 电解质 溶液中, $\mathrm{PMA}_{50} @ \mathrm{CNTs}$ 电极材料在扫速为 $10 \mathrm{mV} / \mathrm{s}$ 时 表现出最大比容量 $(511.7 \mathrm{~F} / \mathrm{g})$, 同时在 $2 \mathrm{~A} / \mathrm{g}$ 电流密 度下获得的最大能量密度达 $66.8 \mathrm{Wh} / \mathrm{kg}$, 相应的功 率密度为 $1000 \mathrm{~W} / \mathrm{kg}$ 。在 $5 \mathrm{~A} / \mathrm{g}$ 电流密度下, 恒电流 充放电循环 1000 次后，比容量无任何衰减，反而有 $2 \%$ 的增加。因此, $\mathrm{PMA}_{50} @ \mathrm{CNTs}$ 复合物有望成为下 一代高性能超级电容器的理想电极材料。

\section{参考文献:}

[1] NAKAMURA O, KODAMA T, OGINO I, et al. High-conducting solid proton conductors: dodecamolybdophosphoric acid and dodecatungstophosphoric acid crystals. Chemistry Letters, 1979, 1(1): 17-18.

[2] BURAKOV V S, BUTSEN A V, BRUSER V, et al. Synthesis of tungsten carbide nanopowder via submerged discharge method. Journal of Nanoparticle Research, 2008, 10(5): 881-886.

[3] CLAIRE C C, SEBASTIENS, LAURENT R. Photocatalysis with polyoxometalates associated to porphyrins under visible light: an application of charge transfer in electrostatic complexes. Journal of Physical Chemistry A, 2010, 114(22): 6394-6400.

[4] DECKER B, HARTMANNTHOMPSON C, CARVER P I, et al. Multilayer sulfonated polyhedral oligosilsesquioxane (s-poss)sulfonated polyphenylsulfone (S-PPSU) composite proton exchange membranes. Chemistry of Materials, 2009, 22(3): 942-948.

[5] POPE M T, MULLER A. Polyoxometalate chemistry: an old field with new dimensions in several disciplines. AngewandteChemie 
International Edition, 1991, 30(1): 34-48.

[6] SU Y, LIU C, SHAN Y, et al. Promotion effect of molybdophorsphoric acid on oxygenreduction for cathode of DMFC. Chem. J. Chin. Univ., 2005, 26(6): 1114-1117.

[7] GOTZ M, WENDT H. Binary and ternary anode catalyst formulations including the elements W, Sn and Mo for PEMFCs operated on methanol or reformate gas. ElectrochimicaActa, 1998, 43(24): 3637-3644

[8] HUANG X L, LIN Z, LIAN X Y, et al. Preparation and electrocatalytic properties of $\mathrm{Pd} / \mathrm{PMo}_{12}-\mathrm{GN}$ composite towards formic acid oxidation. Journal of Inorganic Materials, 2014, 29(7): 722-728.

[9] AND E C, GOMEZGARCIA C J. Polyoxometalate-based molecular materials. Chemical Reviews, 1998, 98(1): 273-296.

[10] WON B K, VOITL T, RODRIGUEZ R G J, et al. Powering fuel cells with $\mathrm{CO}$ via aqueous polyoxometalates and gold catalysts. Science, 2004, 305(5688): 1280-1283.

[11] LIU S, KURTH D G, VOLKMER D. Polyoxometalates as pH-sensitive probes in self-assembled multilayers. Chemical Communications, 2002, 9(9): 976-977.

[12] LIU S L, DIRK V, KURTH D G. Smart polyoxometalate-based nitrogen monoxide sensors. Analytical Chemistry, 2004, 76(15): 4579-4582.

[13] DOUVAS A M, ELENI M, NIKOS G, et al. Polyoxometalate-based layered structures for charge transport control in molecular devices. ACS Nano, 2008, 2(2): 733-742.

[14] ELEFTHERIOS K, DOUVAS A M, DIMITRIS V, et al. Molecular storage elements for proton memory devices. Advanced Materials, 2008, 20(23): 4568-4574.

[15] CORONADO E, GIMENEZ S C, GOMEZ G C J. Recent advances in polyoxometalate-containing molecular conductors. Coordination Chemistry Reviews, 2005, 249(17): 1776-1796.

[16] WANG X L, ZHANG H , WANG E B , et al. Phosphomolybdate-polypyrrole composite bulk-modified carbon paste electrode for a hydrogen peroxide amperometric sensor. Mater. Lett. , 2004, 58(10): 1661-1664.

[17] LIU Z, WU Q Y, SONG X L, et al. Solid high-proton conductors based on heteropoly acids. Progress in Chemistry, 2009, 21(5): 982-989.

[18] QI W, LIU W, LIU S, et al. Heteropoly acid/carbon nanotube hybrid materials as efficient solid-acid catalysts. Chemcatchem, 2014, 6(9): 2613-2620.

[19] WEN S, GUAN W, KAN Y, et al. Theoretical insights into $\left[\mathrm{PMo}_{12} \mathrm{O}_{40}\right]^{3-}$ grafted on single-walled carbon nanotubes. Physical Chemistry Chemical Physics, 2013, 15(23): 9177-9185.

[20] AVCI A K, TRIMM D L, KARAKAYA M. Microreactor catalytic combustion for chemicals processing. Catalysis Today, 2010, 155(1/2): 66-74.

[21] QIN L C, ZHAO X, HIRAHARA K, et al. The smallest carbon nanotube. Nature, 2000, 408(6808): 95-120.

[22] MOORE V C, STRANO M S, HAROZ E H, et al. Individually suspended single-walled carbon nanotubes in various surfactants. Nano Letters, 2003, 3(10): 1379-1382.

[23] AND Y S, THOMAS E L. High-concentration dispersion of single-wall carbon nanotubes. Macromolecules, 2004, 37(13): 4815-4820.

[24] OU Y Y, HUANG M H. High-density assembly of gold nanoparti- cles on multiwalled carbon nanotubes using 1-pyrenemethylamine as interlinker. Journal of Physical Chemistry B, 2006, 110(5): 2031-2036.

[25] TOMA F M, SARTOREL A, IURLO M, et al. Efficient water oxidation at carbon nanotube-polyoxometalateelectrocatalytic interfaces. Nature Chemistry, 2010, 2(10): 826-831.

[26] LEE H, RHO J, MESSERSMITH P B. Facile conjugation of biomolecules onto surfaces via mussel adhesive protein inspired coatings. Advanced Materials, 2009, 21(4): 431-434.

[27] DALSIN J L, HU B H, AND B P L, et al. Mussel adhesive protein mimetic polymers for the preparation of nonfouling surfaces. Journal of the American Chemical Society, 2003, 125(125): 4253-4258.

[28] LEE H, DELlatorE S M, MILLER W M. Mussel-inspired surface chemistry for multifunctional coatings. Science, 2007, 318(5849): 426-430

[29] XI Z Y, XU Y Y, ZHU L P, et al. A facile method of surface modification for hydrophobic polymer membranes based on the adhesive behavior of poly(DOPA) and poly(dopamine). Journal of Membrane Science, 2009, 327(1): 244-253.

[30] FEI B, QIAN B, YANG Z, et al. Coating carbon nanotubes by spontaneous oxidative polymerization of dopamine. Carbon, 2008, 46(13): 1795-1797.

[31] SING K S W. Reporting physisorption data for gas/solid systems with special reference to the determination of surface area and porosity. Pure \& Applied Chemistry, 1982, 54(11): 2201-2218.

[32] LIU C, YU Z, NEFF D, et al. Graphene-based supercapacitor with an ultrahigh energy density. Nano Letters, 2010, 10(12): 4863-4868.

[33] ZHAO X, ZHU J, LING L, et al. Enhanced electroactivity of pdnanocrystals supported on $\mathrm{H}_{3} \mathrm{PMo}_{12} \mathrm{O}_{40}$ /carbon for formic acidelectrooxidation. J. Power Sources, 2012, 210(4): 392-396.

[34] BURZIO L A, WAITE J H. Cross-linking in adhesive quinoproteins: studies with model decapeptides. Biochemistry, 2000, 39(36): 11147-11153.

[35] 齐明立. 含 Keggin 结构磷钼酸盐杂化材料的合成、晶体结构与 性质研究. 哈尔滨: 哈尔滨师范大学硕士学位论文, 2013.

[36] SJOSTROM H, STAFSTROM S, BOMAN M, et al. Superhard and elastic carbon nitride thin films having fullerenelike microstructure. Physical Review Letters, 1995, 75(7): 1336-1339.

[37] TAN K L, WOON L L, WONG H K, et al. Surface modification of plasma-pretreated poly(tetrafluoroethylene) films by graft copolymerization. Macromolecules, 1993, 26(11): 2832-2836.

[38] III J R F, KUO M C, TURNER J A, et al. The use of the heteropoly acids, $\mathrm{H}_{3} \mathrm{PMo}_{12} \mathrm{O}_{40}$ and $\mathrm{H}_{3} \mathrm{PW}_{12} \mathrm{O}_{40}$, for the enhanced electrochemical oxidation of methanol for direct methanol fuel cells. ElectrochimicaActa, 2008, 53(14): 4927-4933.

[39] SUPPES G M, DEORE B A, FREUND M S. Porous conducting polymer/heteropolyoxometalate hybrid material for electrochemicalsupercapacitor applications. Langmuir, 2008, 24(3): 1064-1069.

[40] WU Z S, SUN Y, TAN Y Z, et al. Three-dimensional graphenebased macro- and mesoporous frameworks for high-performance electrochemical capacitive energy storage. Journal of the American Chemical Society, 2012, 134(48): 19532-19535.

[41] LIN T, CHEN I W, LIU F, et al. Nitrogen-doped mesoporous carbon of extraordinary capacitance for electrochemical energy storage. Science, 2015, 350(6267): 1508-1513. 